\title{
On oscillatory behavior of two-dimensional time scale systems
}

\section{Özkan Öztürk* (1)}

\section{"Correspondence:}

ozkanozturk.mst@gmail.com

Department of Mathematics,

Faculty of Arts and Sciences,

Giresun University, Giresun, Turkey

\begin{abstract}
This paper deals with long-time behaviors of nonoscillatory solutions of a system of first-order dynamic equations on time scales. Some well-known fixed point theorems and double improper integrals are used to prove the main results.
\end{abstract}

MSC: 34N05; 39A10; 39A 13

Keywords: nonoscillation; long-time behavior; two-dimensional system; asymptotic properties; time scale systems

\section{Introduction}

In this paper, we consider the following system:

$$
\left\{\begin{array}{l}
x^{\Delta}(t)=a(t) f(y(t)), \\
y^{\Delta}(t)=b(t) g(x(t)),
\end{array}\right.
$$

where $a, b \in C_{r d}\left(\left[t_{0}, \infty\right)_{\mathbb{T}}, \mathbb{R}^{+}\right)$, and $f$ and $g$ are nondecreasing functions such that $u f(u)>0$ and $u g(u)>0$ for $u \neq 0$. The time scale theory is initiated by the German mathematician S. Hilger in his PhD thesis in 1988. His purpose was to unify continuous and discrete analysis and extend the results in one theory. A time scale, denoted by $\mathbb{T}$, is a nonempty closed subset of real numbers, and some examples are the set of real numbers $\mathbb{R}$, the set of integers $\mathbb{Z}, h \mathbb{Z}$ for $h>0$, and $q^{\mathbb{N}_{0}}$ for $q>1$. In this paper, we assume that $\mathbb{T}$ is unbounded above and that $(x, y)$ is a proper solution of system (1); see [1]. We call $(x, y)$ a proper solution if it is defined on $\left[t_{0}, \infty\right)_{\mathbb{T}}$ and $\sup \left\{|x(s)|,|y(s)|: s \in[t, \infty)_{\mathbb{T}}\right\}>0$ for $t \geq t_{0}$. A solution $(x, y)$ is called nonoscillatory if $x$ and $y$ are both nonoscillatory, that is, eventually of one sign. Otherwise, it is said to be oscillatory. For more information about the time scale theory, we refer the interested readers to the books [2, 3] by Bohner and Peterson, respectively.

This paper helps readers to understand the significance of the nonoscillation theory in a more general context. In fact, nonoscillation plays a very important role in understanding the long-time behavior of solutions of a system for several reasons, for example, stability and control theories. Two-dimensional systems of first-order equations have several reallife applications in engineering. For example, Bartolini et al. [4-6] considered a secondorder system in order to control uncertain nonlinear systems by some control techniques, for example, sliding mode and approximate linearization. In addition to nonoscillatory

(c) The Author(s) 2018. This article is distributed under the terms of the Creative Commons Attribution 4.0 International License (http://creativecommons.org/licenses/by/4.0/), which permits unrestricted use, distribution, and reproduction in any medium, provided you give appropriate credit to the original author(s) and the source, provide a link to the Creative Commons license, and indicate if changes were made. 
solutions of second-order systems, periodic and subharmonic solutions were also considered in [7-9], and important results were obtained.

If $\mathbb{T}=\mathbb{R}$, then system (1) turns out to be the system of first-order differential equations, and this system was considered by Li [10]. Also, different versions of system (1) when $\mathbb{T}=\mathbb{Z}$ were considered by Cheng, H. J. Li, and Patula [11], Agarwal, W. T. Li, and Pang [12], and Cecchi, Došlá, and Marini [13, 14]. The time-scale versions of system (1) were studied by Öztürk and Akın [15-18]. For example, Öztürk, Akın, and Tiryaki [16] considered a different version of system (1), known as the Emden-Fowler dynamical system in the literature,

$$
\left\{\begin{array}{l}
x^{\Delta}(t)=\left(\frac{1}{a(t)}\right) \frac{1}{\alpha}|y(t)|^{\frac{1}{\alpha}} \operatorname{sgn} y(t), \\
y^{\Delta}(t)=-b(t)\left|x^{\sigma}(t)\right|^{\beta} \operatorname{sgn} x^{\sigma}(t),
\end{array}\right.
$$

where $\alpha, \beta>0$ and $a, b \in C_{r d}\left(\left[t_{0}, \infty\right)_{\mathbb{T}}, \mathbb{R}^{+}\right)$, and investigated nonosillatory behaviors by using $\alpha$ and $\beta$ relations and some improper integrals. The Emden-Fowler dynamical system has several applications in astrophysics, gas dynamics and fluid mechanics, relativistic mechanics, nuclear physics, and chemically reacting systems; see [19-26].

Supposing that $S$ is the set of all nonoscillatory solutions of system (1), we can easily show that any nonoscillatory solution $(x, y)$ of system (1) belongs to one of the following classes:

$$
\begin{aligned}
& S^{+}:=\{(x, y) \in S: x y>0 \text { eventually }\}, \\
& S^{-}:=\{(x, y) \in S: x y<0 \text { eventually }\} .
\end{aligned}
$$

In this paper, we only focus on $S^{-}$since the existence in $S^{+}$is examined by Öztürk [1]. Without loss of generality, we assume that $x>0$ eventually, and proofs are similar when $x<0$ eventually. By a solution we mean a nonoscillatory solution.

The structure of the paper is as follows. In Section 1, we provide the existence and nonexistence of solutions of system (1). In Section 2, we present examples for our theoretical claims, and finally, we give a conclusion in the last section. For simplicity, we set

$$
A\left(t_{0}, t\right)=\int_{t_{0}}^{t} a(s) \Delta s \quad \text { and } \quad B\left(t_{0}, t\right)=\int_{t_{0}}^{t} b(s) \Delta s .
$$

Suppose that $(x, y)$ is a nonoscillatory solution of system (1) such that $x>0$ eventually. Then by the first and second equations of system (1) we have that $x$ is positive decreasing and $y$ is negative increasing eventually. So we have that $x \rightarrow c$ or $x \rightarrow 0$ and $y \rightarrow-d$ or $y \rightarrow 0$ for $0<c<\infty$ and $0<d<\infty$. So in view of our discussion, we can get the following subclasses of $S^{-}$:

$$
\begin{aligned}
& S_{B, B}^{-}=\left\{(x, y) \in S^{-}: \lim _{t \rightarrow \infty} x(t)=c, \lim _{t \rightarrow \infty} y(t)=-d\right\}, \\
& S_{B, 0}^{-}=\left\{(x, y) \in S^{-}: \lim _{t \rightarrow \infty} x(t)=c, \lim _{t \rightarrow \infty} y(t)=0\right\}, \\
& S_{0, B}^{-}=\left\{(x, y) \in S^{-}: \lim _{t \rightarrow \infty} x(t)=0, \lim _{t \rightarrow \infty} y(t)=-d\right\}, \\
& S_{0,0}^{-}=\left\{(x, y) \in S^{-}: \lim _{t \rightarrow 0} x(t)=0, \lim _{t \rightarrow 0} y(t)=0\right\} .
\end{aligned}
$$


To show the existence, we use the following fixed point theorems known as Schauder's fixed point theorem and Knaster's fixed point theorem in the literature, see [27, Theorem 2.A] and [28], respectively.

Theorem 1 Let $X$ be a Banach space such that $Y$ is a closed, nonempty, convex, and bounded subset of $X$. Suppose also $F: Y \rightarrow Y$ is a compact operator. Then $F$ has a fixed point.

Theorem 2 If $(Y, \leq)$ is a complete lattice and $F: Y \rightarrow Y$ is order-preserving, then $F$ has a fixed point. As a matter of fact, the set of fixed points of $F$ is a complete lattice.

\section{Main results}

\subsection{Existence in $S^{-}$}

In this section, we provide the existence and nonexistence of nonoscillatory solutions of system (1) with the help of the following improper integrals under the monotonicity condition on $f$ and $g$ :

$$
\begin{aligned}
& I_{1}=\int_{t_{0}}^{\infty} a(t) f\left(k-l \int_{t}^{\infty} b(s) \Delta s\right) \Delta t, \\
& I_{2}=\int_{t_{0}}^{\infty} b(t) g\left(m \int_{t}^{\infty} a(s) \Delta s\right) \Delta t,
\end{aligned}
$$

where $k, l$, and $m$ are some constants.

Theorem 3 Let $B\left(t_{0}, \infty\right)<\infty$. Then $S_{B, B}^{-} \neq \emptyset$ if and only if $I_{1}<\infty$, provided that $k<0$ and $l>0$.

Proof Suppose $S_{B, B}^{-} \neq \emptyset$. Then there exists a solution $(x, y) \in S_{B, B}^{-}$such that $x>0, y<0$, $x(t) \rightarrow c_{1}$ and $y(t) \rightarrow-d_{1}$ as $t \rightarrow \infty$ for $0<c_{1}<\infty$ and $0<d_{1}<\infty$. By the monotonicity of $g$ and integrating the second equation of system (1) from $t$ to $\infty$, we obtain

$$
\begin{aligned}
y(t) & =y(\infty)-\int_{t}^{\infty} b(s) g(x(s)) \Delta s \\
& \leq-d_{1}-l \int_{t}^{\infty} b(s) \Delta s, \quad \text { where } l=g\left(c_{1}\right) .
\end{aligned}
$$

Since $x$ is bounded, integrating the first equation from $t_{1}$ to $t$ and using (2) result in

$$
\begin{aligned}
c_{1} & \leq x(t)=x\left(t_{1}\right)+\int_{t_{1}}^{t} a(s) f(y(s)) \Delta s \\
& \leq x\left(t_{1}\right)+\int_{t_{1}}^{t} a(s) f\left(-d_{1}-l \int_{s}^{\infty} b(u) \Delta u\right) \Delta s \leq x\left(t_{1}\right), \quad t \geq t_{1} .
\end{aligned}
$$

This implies that $I_{1}<\infty$ as $t \rightarrow \infty$, where $-d_{1}=k$.

Conversely, suppose that $I_{1}<\infty$. Then there exist $t_{1} \geq t_{0}$ and $k<0, l>0$ such that

$$
\int_{t_{0}}^{\infty} a(t) f\left(k-l \int_{t}^{\infty} b(s) \Delta s\right) \Delta t>-\frac{1}{2}
$$


where $l=g\left(\frac{3}{2}\right)$. Let $X$ be the set of all continuous and bounded real-valued functions $x(t)$ on $\left[t_{1}, \infty\right)_{\mathbb{T}}$ with the supremum norm $\sup _{t \geq t_{1}}|x(t)|$, which implies that $X$ is a Banach space [29]. Let $Y$ be the subset of $X$ defined as

$$
Y:=\left\{x(t) \in X: 1 \leq x(t) \leq \frac{3}{2}, t \geq t_{1}\right\} .
$$

It can be shown that $Y$ satisfies the conditions of Theorem 1. Let us define the operator $F: Y \rightarrow X$ by

$$
(F x)(t)=1-\int_{t}^{\infty} a(s) f\left(k-\int_{s}^{\infty} b(u) g(x(u)) \Delta u\right) \Delta s .
$$

First, we need to show that $F$ is a mapping into itself, thats is, $F: Y \rightarrow Y$. Indeed,

$$
1 \leq(F x)(t) \leq 1-\int_{t}^{\infty} a(s) f\left(k-g\left(\frac{3}{2}\right) \int_{t_{1}}^{s} b(u) \Delta u\right) \Delta s \leq \frac{3}{2}
$$

because $x \in Y$ and (3) holds. Next, let us verify that $F$ is continuous on $Y$. To this end, let $x_{n}$ be a sequence in $Y$ such that $x_{n} \rightarrow x$, where $x \in Y=\bar{Y}$. Then

$$
\begin{aligned}
& \left|\left(F x_{n}\right)(t)-(F x)(t)\right| \\
& \quad \leq \int_{t}^{\infty} a(s)\left|f\left(k-\int_{s}^{\infty} b(u) g\left(x_{n}(u)\right) \Delta u\right)-f\left(k-\int_{s}^{\infty} b(u) g(x(u)) \Delta u\right)\right| \Delta s .
\end{aligned}
$$

Therefore, the continuity of $f$ and $g$ and the Lebesgue dominated convergence theorem gives $F x_{n} \rightarrow F x$ as $n \rightarrow \infty$, which implies that $F$ is continuous on $Y$. Finally, we prove that $F Y$ is equibounded and equicontinuous, that is, relatively compact. Because

$$
\begin{aligned}
0 & <(F x)^{\Delta}(t)=a(t) f\left(k-\int_{t}^{\infty} b(u) g(x(u)) \Delta u\right) \\
& \leq a(t) f\left(k-l \int_{t_{1}}^{t} b(u) \Delta u\right)<\infty,
\end{aligned}
$$

we have that $F x$ is relatively compact. Hence, Theorem 1 implies that there exists $\bar{x} \in Y$ such that $\bar{x}=F \bar{x}$. Thus, we have $\bar{x}>0$ eventually and $\bar{x}(t) \rightarrow 1$ as $t \rightarrow \infty$. Also

$$
\bar{x}^{\Delta}(t)=(F \bar{x})^{\Delta}(t)=a(t) f\left(k-\int_{t}^{\infty} b(u) g(\bar{x}(u)) \Delta u\right), \quad t \geq t_{1} .
$$

Letting

$$
\bar{y}(t)=k-\int_{t}^{\infty} b(u) g(\bar{x}(u)) \Delta u<0, \quad t \geq t_{1},
$$

and taking the derivative of (5) give $\bar{y}^{\Delta}(t)=b(t) g(\bar{x}(t))$. So, we conclude that $(\bar{x}, \bar{y})$ is a nonoscillatory solution of system (1). Finally, taking the limit of equation (5) results in $\bar{y}(t) \rightarrow k$. Therefore, we get $S_{B, B}^{-} \neq \emptyset$.

Theorem 4 Suppose $B\left(t_{0}\right)<\infty . S_{B, 0}^{-} \neq \emptyset$ if and only if $I_{1}<\infty$, where $k=0$ and $l>0$. 
Proof For necessity, suppose that there exists a solution $(x, y)$ in $S_{B, 0}^{-}$such that $x$ is positive and $y$ is negative eventually. By the definition of $S_{B, 0}^{-}$we have that $x(t)$ tends to a positive finite constant $c_{1}$ and $y(t)$ tends to 0 as $t \rightarrow \infty$. Since $x$ is bounded, there exists $t_{1} \geq t_{0}$ such that $c_{1} \leq x(t) \leq x\left(t_{1}\right), t \geq t_{1}$. Taking the integral of the last equation of (1) from $t$ to $\infty$, we have

$$
y(t) \leq-g\left(c_{1}\right) \int_{t}^{\infty} b(s) \Delta s
$$

Then by the monotonicity of $f$, integrating the first equation of system (1), we get

$$
c_{1} \leq x(t)=x\left(t_{1}\right)+\int_{t_{1}}^{t} a(s) f(y(s)) \Delta s \leq x\left(t_{1}\right)
$$

The latter inequality and (6) imply that

$$
c_{1}-x\left(t_{1}\right) \leq \int_{t_{1}}^{t} a(s) f\left(-g\left(c_{1}\right) \int_{t_{1}}^{t} b(u) \Delta u\right) \Delta s \leq 0 .
$$

Therefore, as $t \rightarrow \infty$, the assertion follows for $l=g\left(c_{1}\right)$.

For sufficiency, suppose $I_{1}<\infty$ holds for $k=0$ and $l>0$. Then choose $t_{1} \geq t_{0}$ and $l>0$ such that

$$
-\frac{1}{2}<\int_{t_{1}}^{\infty} a(t) f\left(-l \int_{t_{1}}^{t} b(s) \Delta s\right) \Delta t<0
$$

where $l=g(1)$. Let $X$ be the partially ordered space of continuous functions on $\left[t_{0}, \infty\right)_{\mathbb{T}}$ with the norm $\sup _{t \geq t_{1}}|x(t)|$ and pointwise ordering $\leq$. Let $Y$ be the subset of $X$ defined by

$$
Y=\left\{x \in X: \frac{1}{2} \leq x(t) \leq 1 t \geq t_{1}\right\}
$$

and define the operator $F: Y \rightarrow X$ by

$$
(F x)(t)=\frac{1}{2}-\int_{t}^{\infty} a(s) f\left(-\int_{s}^{\infty} b(u) g(x(u)) \Delta u\right) \Delta t, \quad t \geq t_{1} .
$$

It can be easily verified that $\inf \Omega_{1} \in Y$ and $\sup \Omega_{1} \in Y$ for any subset $\Omega_{1}$ of $Y$, which implies that $(Y, \leq)$ is a complete lattice. First, we show that $F: Y \rightarrow Y$ is a mapping into itself. Since

$$
\frac{1}{2} \leq(F x)(t) \leq \frac{1}{2}-\int_{t}^{\infty} a(s) f\left(-g(1) \int_{s}^{\infty} b(u) \Delta u\right) \Delta t \leq 1, \quad t \geq t_{1},
$$

we have that $F: Y \rightarrow Y$. Showing that $F$ is an increasing mapping can be done by the definition, that is, if $x_{1} \leq x_{2}$, then $F x_{1} \leq F x_{2}$. Then by Theorem 2 there exists a function $\bar{x} \in \Omega$ such that $\bar{x}=F \bar{x}$. Therefore, we obtain

$$
(F \bar{x})^{\Delta}(t)=a(t) f\left(-\int_{t}^{\infty} b(u) g(\bar{x}(u)) \Delta u\right), \quad t \geq t_{1} .
$$


Letting

$$
\bar{y}(t)=-\int_{t}^{\infty} b(u) g(\bar{x}(u)) \Delta u,
$$

we have that $\bar{y}^{\Delta}(t)=b(t) g(\bar{x}(t))$ and $(\bar{x}, \bar{y})$ is a solution of system (1) such that $\bar{x}$ tends to $\frac{1}{2}$ and $\bar{y}$ tends to zero, that is, $S_{B, 0}^{-} \neq \emptyset$. This finishes the proof.

Theorem 5 Suppose $A\left(t_{0}\right)<\infty . S_{0, B}^{-} \neq \emptyset$ if and only if $I_{2}<\infty$ for $m>0$.

Proof The necessary condition can be proven similarly to the previous theorems. For sufficiency, suppose that $I_{2}<\infty$ for $m>0$. Let $X$ be the partially ordered space of continuous functions with the supremum norm $\sup _{t \geq t_{1}}|y(t)|$ and pointwise ordering $\leq$. Define the subset $Y$ of $X$ as

$$
Y:=\left\{y \in X:-1 \leq y(t) \leq-\frac{1}{2} t \geq t_{1}\right\}
$$

and the operator $F: Y \rightarrow X$ by

$$
(F y)(t)=-\frac{1}{2}-\int_{t}^{\infty} b(s) g\left(-\int_{s}^{\infty} a(u) f(y(u)) \Delta u\right) \Delta s, \quad t \geq t_{1} .
$$

As claimed in the previous theorem, it can be verified that $(Y, \leq)$ is a complete lattice and $F$ is an increasing mapping. Therefore, let us show that $F$ is a mapping into itself. Indeed,

$$
-1 \leq-\frac{1}{2}-\int_{t}^{\infty} b(s) g\left(-f\left(-\frac{1}{2}\right) \int_{s}^{\infty} a(u) \Delta u\right) \Delta t \leq(F y)(t) \leq-\frac{1}{2}, \quad t \geq t_{1} .
$$

Then by Theorem 2 there exists a function $\bar{y} \in Y$ such that $\bar{y}=F \bar{y}$. By taking the derivative of $F \bar{y}$ we have

$$
(F \bar{y})^{\Delta}(t)=b(t) g\left(-\int_{t}^{\infty} a(u) f(\bar{y}(u)) \Delta u\right), \quad t \geq t_{1} .
$$

Setting

$$
\bar{x}(t)=-\int_{t}^{\infty} a(u) f(\bar{y}(u)) \Delta u>0, \quad t \geq t_{1}
$$

gives us that $\bar{x}^{\Delta}(t)=a(t) f(\bar{y}(t))$ and $(\bar{x}, \bar{y})$ is a nonoscillatory solution of system in $S_{0, B}^{-}$, which concludes the proof.

Theorem 6 Suppose $A\left(t_{0}\right)<\infty$. $S_{0,0}^{-} \neq \emptyset$ if $I_{1}<\infty$ and $I_{2}=\infty$ for $k=0, l<0$, and $m>0$, provided that $f$ is odd.

Proof Suppose that $I_{1}<\infty$ and $I_{2}=\infty$. Then there exists $t_{1} \geq t_{0}$ such that

$$
\int_{t_{1}}^{\infty} a(s) f\left(-l \int_{s}^{\infty} b(u) \Delta u\right) \Delta s<1
$$


and

$$
\int_{t_{1}}^{\infty} b(s) g\left(m \int_{s}^{\infty} a(u) \Delta u\right) \Delta s>\frac{1}{2}
$$

for $t \geq t_{1}, l=-g(1)$. Let $X$ be the space as in the proof of Theorem 4. Let $Y$ be the subset of $X$ given by

$$
Y:=\left\{x \in X: c_{1} \int_{t}^{\infty} a(s) \Delta s \leq x(t) \leq 1 t \geq t_{1}\right\},
$$

where $c_{1}=f\left(\frac{1}{2}\right)$. Define the operator $H: Y \rightarrow X$ by

$$
(H x)(t)=\int_{t}^{\infty} a(s) f\left(\int_{s}^{\infty} b(u) g(x(u)) \Delta u\right) \Delta t, \quad t \geq t_{1} .
$$

As shown in the proof of Theorem 4, we can show that $(Y, \leq)$ is a complete lattice and $H$ is an increasing mapping. Next, let us justify that $H: Y \rightarrow Y$. Indeed,

$$
(H x)(t) \leq \int_{t}^{\infty} a(s) f\left(g(1) \int_{s}^{\infty} b(u) \Delta u\right) \Delta t \leq 1, \quad t \geq t_{1},
$$

and

$$
\begin{aligned}
(H x)(t) & \geq \int_{t}^{\infty} a(s) f\left(\int_{s}^{\infty} b(u) g\left(c_{1} \int_{u}^{\infty} a(v) \Delta v\right) \Delta u\right) \Delta s \\
& \geq f\left(\frac{1}{2}\right) \int_{t}^{\infty} a(s) \Delta s,
\end{aligned}
$$

where $c_{1}=m$. Then by Theorem 2 there exists a function $\bar{x} \in Y$ such that $\bar{x}=H \bar{x}$. By taking the derivative of $H \bar{x}$ and using the fact that $f$ is odd, we have

$$
(H \bar{x})^{\Delta}(t)=a(t) f\left(-\int_{t}^{\infty} b(u) g(\bar{x}(u)) \Delta u\right), \quad t \geq t_{1} .
$$

Setting

$$
\bar{y}(t)=-\int_{t}^{\infty} b(u) g(\bar{x}(u)) \Delta u
$$

yields that $\bar{y}^{\Delta}(t)=b(t) g(\bar{x}(t))$ and $(\bar{x}, \bar{y})$ is a solution of system (1) in $S_{0,0}^{-}$, that is, $\bar{x}$ and $\bar{y}$ both tend to zero.

\subsection{Nonexistence in $\mathrm{S}^{-}$}

In this section, we relax the monotonicity condition on $f$ and $g$ and assume that there exist positive constants $F$ and $G$ such that

$$
\frac{f(u)}{u} \geq F \quad \text { and } \quad \frac{g(u)}{u} \geq G \quad \text { for } u \neq 0 .
$$

To show the nonexistence of nonoscillatory solutions in $S^{-}$, note that we have already had the nonexistence of such solutions in $S_{B, B}^{-}, S_{B, 0}^{-}, S_{0, B}^{-}$by using the monotonicity condition in 
the previous section. Now, we show similar results by relaxing the monotonicity condition of $f$ and $g$. Let

$$
\begin{aligned}
& I_{3}=\int_{t_{0}}^{\infty} b(t)\left(\int_{t}^{\infty} a(s) \Delta s\right) \Delta t, \\
& I_{4}=\int_{t_{0}}^{\infty} a(t)\left(\int_{t}^{\infty} b(s) \Delta s\right) \Delta t .
\end{aligned}
$$

Theorem 7 Let $B\left(t_{0}, \infty\right)<\infty$. If $I_{3}=\infty$, then $S_{B, B}^{-}=\emptyset$.

Proof The proof is by contradiction. So assume that $S_{B, B}^{-} \neq \emptyset$. Then there exists a nonoscillatory solution $(x, y)$ and $t_{1} \geq t_{0}$ such that $x(t)>0$ and $y(t)<0$ for $t \geq t_{1}$. Also, since $x$ is decreasing and $y$ is increasing eventually, we have $c_{1} \leq x(t) \leq c_{2}$ and $-d_{1} \leq y(t) \leq-d_{2}$ for $t \geq t_{1}$. Integrating the first equation of system (1) from $t$ to $\infty$, by condition (8) we have

$$
x(t) \geq c_{1}-F \int_{t}^{\infty} a(s) y(s) \Delta s, \quad t \geq t_{1} .
$$

Integrating the second equation from $t_{1}$ to $t$, condition (8), and inequality (9) yield

$$
\begin{aligned}
y(t) & \geq y\left(t_{1}\right)-F G \int_{t_{1}}^{t} b(s)\left(\int_{s}^{\infty} a(u) y(u) \Delta u\right) \Delta s \\
& \geq y\left(t_{1}\right)+F G d_{2} \int_{t_{1}}^{t} b(s)\left(\int_{s}^{\infty} a(u) \Delta u\right) \Delta s, \quad t \geq t_{1} .
\end{aligned}
$$

Therefore, as $t \rightarrow \infty$, we have a contradiction to the fact that $y<0$ eventually. This completes the proof.

Theorem 8 Suppose $B\left(t_{0}, \infty\right)<\infty$. If $I_{4}=\infty$, then $S_{B, 0}^{-}=\emptyset$.

Proof Assume the contrary. Then there exists a nonoscillatory solution $(x, y)$ in $S_{B, 0}^{-}$and $t \geq t_{1}$ such that $x(t)>0, y(t)<0$, and $c_{1} \leq x(t) \leq c_{2}$ for $t \geq t_{1}$. Integrating the second equation of system (1) from $t$ to $\infty$ and using condition (8), we have

$$
y(t) \leq-G \int_{t}^{\infty} b(s) x(s) \Delta s, \quad t \geq t_{1}, \text { where } G>0 .
$$

Next, integrating the second equation of system (1) from $t_{1}$ to $t$, inequality (10), and the fact that $x$ is bounded yield us

$$
c_{1}-x\left(t_{1}\right) \leq \int_{t_{1}}^{t} a(s) f(y(s)) \Delta s \leq-F G \int_{t_{1}}^{t} a(s)\left(\int_{s}^{\infty} b(u) \Delta u\right) \Delta s<0, \quad t \geq t_{1} .
$$

Hence, we have a contradiction to $I_{4}=\infty$ as $t \rightarrow \infty$, which finishes the proof.

The following theorem can be proven similarly to the previous theorems.

Theorem 9 Let $A\left(t_{0}, \infty\right)<\infty$. If $I_{3}=\infty$, then $S_{0, B}^{-}=\emptyset$. 


\section{Examples}

Making a statement without examples can make the results muddy, whereas examples make results clearer and give more information to readers. Therefore, we give the following examples for validating our claims.

Theorem 10 ([2, Theorem 1.79]) Let $a, b \in \mathbb{T}$ and $f \in \mathrm{C}_{\mathrm{rd}}$. If $[a, b]$ consists of only isolated points, then

$$
\int_{a}^{b} f(t) \Delta t=\sum_{t \in[a, b)_{\mathbb{T}}} \mu(t) f(t) .
$$

Example 1 Let $\mathbb{T}=q^{\mathbb{N}_{0}}, q>1$. Consider the following system:

$$
\left\{\begin{array}{l}
\Delta x_{q}(t)=\frac{1}{q t^{\frac{8}{5}}\left(2 t^{2}+1\right)^{\frac{1}{5}}}(y(t))^{\frac{1}{5}}, \\
\Delta y_{q}(t)=\frac{q+1}{q^{2} t^{2}(t+1)} x(t),
\end{array}\right.
$$

where $\Delta f_{q}(t)=\frac{f^{\sigma}(t)-f(t)}{\mu(t)}$ for $f^{\sigma}(t)=f(\sigma(t)), \sigma(t)=t q, \mu(t)=(q-1) t$, and $t=q^{n}, s=q^{m}$; see [2]. First, we show $B\left(t_{0}, \infty\right)<\infty$ for $t_{0}=1$. Indeed,

$$
\int_{1}^{T} b(t) \Delta t=\sum_{t \in[1, T)_{q} \mathbb{N}_{0}} \frac{q+1}{q^{2} t^{2}(t+1)} \cdot t .
$$

Therefore, as $T \rightarrow \infty$, we have

$$
\frac{q+1}{q^{2}} \sum_{n=1}^{\infty} \frac{}{q^{n}\left(q^{n}+1\right)}<\infty
$$

by the geometric series, that is, $B(1, \infty)<\infty$. Next, let us show that $I_{1}<\infty$ for $k=-1, l=1$. First, note that

$$
\int_{t}^{T} b(s) \Delta s \leq \frac{q+1}{q^{2}} \sum_{s \in[t, T)_{q} \mathbb{N}_{0}} \frac{1}{s^{2}} .
$$

Taking the limit as $T \rightarrow \infty$, we get $B(t, \infty) \leq \frac{1}{q-1} \frac{1}{t^{2}}$. Second,

$$
\begin{aligned}
\int_{1}^{T} a(t) f\left(-1-\int_{t}^{\infty} b(s) \Delta s\right) \Delta t & \geq \int_{1}^{T} a(t)\left(-1-\frac{1}{q-1} \frac{1}{t^{2}}\right) \\
& \geq-\frac{1}{q} \sum_{t \in[1, T)_{q^{\mathbb{N}}}} \frac{1}{t^{\frac{3}{5}}\left(2 t^{2}+1\right)} .
\end{aligned}
$$

As $T \rightarrow \infty$, we have that $I_{1}$ is convergent by the geometric series and comparison theorem. Finally, we can show that $\left(1+\frac{1}{t},-2-\frac{1}{t^{2}}\right)$ is a solution of system (11) such that $x \rightarrow 1$ and $\rightarrow-2$, that is, $S_{B, B}^{-} \neq \emptyset$ by Theorem 3 . 
Example 2 Consider $\mathbb{T}=\mathbb{N}_{0}^{2}=\left\{n^{2}: n \in \mathbb{N}_{0}\right\}$ with the system

$$
\left\{\begin{array}{l}
x^{\Delta}(t)=\frac{1}{t^{\frac{1}{3}}(\sqrt{t}+1)^{2}\left(t^{2}+1\right)^{\frac{1}{3}}}(y(t))^{\frac{1}{3}} \\
y^{\Delta}(t)=\frac{(\sqrt{t}+1)^{4}-t^{2}}{t^{\frac{9}{5}}(\sqrt{t}+1)^{4}(1+2 \sqrt{t})}(x(t))^{\frac{1}{5}}
\end{array}\right.
$$

where $f^{\Delta}(t)=\frac{f(\sigma(t))-f(t)}{\mu(t)}$ for $\sigma(t)=(\sqrt{t}+1)^{2}$ and $\mu(t)=1+2 \sqrt{t}$; see [2]. First, let us show that $A\left(t_{0}, \infty\right)<\infty$, where $t_{0} \geq 1$. We have

$$
\int_{1}^{T} a(t) \Delta t=\sum_{t \in[1, T)_{\mathbb{N}_{0} 2}} \frac{1}{t^{\frac{1}{3}}(\sqrt{t}+1)^{2}\left(t^{2}+1\right)^{\frac{1}{3}}} \cdot(1+2 \sqrt{t}) \leq \sum_{t \in[1, T)_{\mathbb{N}_{0} 2}} \frac{1+2 \sqrt{t}}{t^{2}} .
$$

Since $t=n^{2}$, as $T \rightarrow \infty$, we have

$$
\sum_{n=1}^{\infty} \frac{1+2 n}{n^{4}}<\infty
$$

by the geometric series. Therefore, $A(1, \infty)<\infty$ by the comparison test. Next, we show that $I_{2}<\infty$. Since $A(1, \infty)<\infty$, we have $\int_{t}^{\infty} a(s) \Delta s<\alpha$ for $t \geq 1$ and $0<\alpha<\infty$. Hence,

$$
\begin{aligned}
\int_{1}^{T} b(t) g\left(\int_{t}^{\infty} a(s) \Delta s\right) \Delta t & \leq \alpha \int_{1}^{T} b(t) \Delta t \\
& =\alpha \sum_{t \in[1, T)_{\mathbb{N}_{0}}} \frac{(\sqrt{t}+1)^{4}-t^{2}}{t^{\frac{9}{5}}(\sqrt{t}+1)^{4}(1+2 \sqrt{t})} \cdot(1+2 \sqrt{t}) \\
& \leq \alpha \sum_{t \in[1, T)_{\mathbb{N}_{0} 2}} \frac{1}{t^{\frac{9}{5}}}
\end{aligned}
$$

So, as $T$ tends to infinity, we get

$$
\sum_{n=1}^{\infty} \frac{1}{n^{\frac{18}{5}}}<\infty
$$

that is, $I_{2}<\infty$. Also, it is easy to verify that $\left(\frac{1}{t},-1-\frac{1}{t^{2}}\right)$ is a solution of system (12) in $S^{-}$ such that $x$ tends to zero whereas $y$ tends to -1 , that is, $S_{0, B}^{-} \neq \emptyset$.

\section{Conclusion}

In this paper, we show the existence of solutions of system (1) rather than in advance assuming that there exist solutions. After guaranteeing the existence of such solutions, we examine the long-time behavior of nonoscillatory solutions of system (1). In general, it is not easy to construct an explicit solution for nonlinear systems. Therefore, providing examples with explicit solutions to our system makes the results more interesting and powerful. Tables 1 and 2 summarize the limit behavior of solutions in $S^{-}$by means of the improper integrals. 
Table 1 Existence in $S^{-}$

\begin{tabular}{llll}
\hline$S_{B, B}^{-}$ & $\neq \emptyset$ & $B\left(t_{0}, \infty\right)<\infty$ & $I_{1}<\infty, k<0, I>0$ \\
$S_{B, 0}^{-}$ & $\neq \emptyset$ & $B\left(t_{0}, \infty\right)<\infty$ & $I_{1}<\infty, k=0, I>0$ \\
$S_{0, B}^{-}$ & $\neq \emptyset$ & $A\left(t_{0}, \infty\right)<\infty$ & $I_{2}<\infty, m>0$ \\
$S_{0,0}^{-}$ & $\neq \emptyset$ & $A\left(t_{0}, \infty\right)<\infty$ & $I_{1}<\infty, I_{2}=\infty, k=0, I<0, m>0$ \\
\hline
\end{tabular}

Table 2 Nonexistence in $S^{-}$

\begin{tabular}{llll}
\hline$S_{B, B}^{-}$ & $=\emptyset$ & $B\left(t_{0}, \infty\right)<\infty$ & $I_{3}=\infty$ \\
$S_{0, B}^{-}$ & $=\emptyset$ & $A\left(t_{0}, \infty\right)<\infty$ & $I_{3}=\infty$ \\
$S_{B, 0}^{-}$ & $=\emptyset$ & $B\left(t_{0}, \infty\right)<\infty$ & $I_{4}=\infty$ \\
\hline
\end{tabular}

\section{Competing interests}

The author declares that they have no competing interests.

Authors' contributions

All authors read and approved the final manuscript.

\section{Publisher's Note}

Springer Nature remains neutral with regard to jurisdictional claims in published maps and institutional affiliations.

Received: 25 April 2017 Accepted: 7 January 2018 Published online: 15 January 2018

\section{References}

1. Öztürk, Ö: Classification schemes of nonoscillatory solutions for two-dimensional time scale systems. Math. Inequal. Appl. 20(2), 377-387 (2017)

2. Bohner, M, Peterson, A: Dynamic Equations on Time Scales: An Introduction with Applications. Birkhäuser, Boston (2001)

3. Bohner, M, Peterson, A: Advances in Dynamic Equations on Time Scales. Birkhäuser, Boston (2003)

4. Bartolini, G, Pvdvnowski, P: Approximate linearization of uncertain nonlinear systems by means of continuous control. In: Proc. of the 30th Conference on Decision and Control, Brighton, England (1991)

5. Bartolini, G, Pisano, A, Punta, E, Usai, E: A survey of applications of second-order sliding mode control to mechanical systems. Int. J. Control 76(9-10), 875-892 (2010)

6. Bartolini, G, Ferrara, A, Usai, E: Applications of a sub-optimal discontinuous control algorithm for uncertain second order systems. Int. J. Robust Nonlinear Control 7, 299-319 (1997)

7. Esmailzadeh, E, Mehri, B, Nakhaie, J: Periodic solution of a second order, autonomous, nonlinear system. Nonlinear Dyn. 10, 307-316 (1996)

8. Li, X, Zhang, Z: Periodic solutions for some second order differential equations with singularity. Z. Angew. Math. Phys. 59, 400-415 (2008)

9. Fonda, A, Manásevich, R, Zanolin, F: Subharmonic solutions for some second-order differential equations with singularities. SIAM J. Math. Anal. 24(5), 1294-1311 (1993)

10. Li, WT: Classification schemes for positive solutions of nonlinear differential systems. Math. Comput. Model. 36, 411-418 (2002)

11. Cheng, S, Li, HJ, Patula, WT: Bounded and zero convergent solutions of second order difference equations. J. Math. Anal. Appl. 141, 463-483 (1989)

12. Agarwal, RP, Li, WT, Pang, PYH: Asymptotic behavior of nonlinear difference systems. Appl. Math. Comput. 140, 307-316 (2003)

13. Cecchi, M, Došlá, Z, Marini, M: Unbounded solutions of quasi-linear difference equations. Comput. Math. Appl. 45 1113-1123 (2003)

14. Cecchi, M, Došlá, Z, Marini, M: Positive decreasing solutions of quasi-linear difference equations. Comput. Math. Appl. 42 1401-1410 (2001)

15. Öztürk, Ö, Akın, E: Classification of nonoscillatory solutions of nonlinear dynamic equations on time scales. Dyn. Syst. Appl. 25, 219-236 (2016)

16. Öztürk, Ö, Akın, E, Tiryaki, Ui: On nonoscillatory solutions of Emden-Fowler dynamic systems on time scales. Filomat 31(6), 1529-1541 (2017)

17. Öztürk, Ö, Akın, E: Nonoscillation criteria for two dimensional time scale systems. Nonauton. Dyn. Syst. 3, 1-13 (2016)

18. Öztürk, Ö, Akın, E: On nonoscillatory solutions of two dimensional nonlinear delay dynamical systems. Opusc. Math. $36,5(2016)$

19. Thompson, W (Kelvin, L): On the convective equilibrium of temperature in the atmosphere. Manchester Philos. Soc. Proc. 2, 170-176 (1860-62); reprint, Math and Phys., Papers by Lord Kelvin, 3, pp. 255-260 (1890)

20. Homerlane, IJ: On the theoretical temperature of the sun under the hypothesis of a gaseous mass maintaining its volume by its internal heat and depending on the laws of gases known to terrestial experiment. Am. J. Sci. Arts 4 , 57-74 (1869-70)

21. Chandrasekhar, S: Introduction to the Study of Steller Structure. University of Chicago Press, Chicago (1939) Chapter 4. (Reprint: Dover, New York, 1957)

22. Chandrasekhar, S: Principles of Stellar Dynamics. University of Chicago Press, Chicago (1942) Chap. V 
23. Fowler, $\mathrm{RH}$ : The form near infinity of real, continuous solutions of a certain differential equation of the second order. Quart. J. Math. 45, 289-350 (1914)

24. Fowler, RH: The solution of Emden's and similar differential equations. Mon. Not. R. Astron. Soc. 91, 63-91 (1930)

25. Fowler, $\mathrm{RH}$ : Some results on the form near infinity of real continuous solutions of a certain type of second order differential equations. Proc. Lond. Math. Soc. 13, 341-371 (1914)

26. Fowler, RH: Further studies of Emden's and similar differential equations. Quart. J. Math. 2, 259-288 (1931)

27. Zeidler, E: Nonlinear Functional Analysis and Its Applications - I: Fixed Point Theorems. Springer, New York (1986)

28. Knaster, B: Un théorème sur les fonctions d'ensembles. Ann. Soc. Pol. Math. 6, 133-134 (1928)

29. Ciarlet, PG: Linear and Nonlinear Functional Analysis with Applications. SIAM, Philadelphia (2013)

\section{Submit your manuscript to a SpringerOpen ${ }^{\circ}$} journal and benefit from:

- Convenient online submission

- Rigorous peer review

- Open access: articles freely available online

- High visibility within the field

- Retaining the copyright to your article

Submit your next manuscript at $\gg$ springeropen.com 